- Original Article

\title{
Trends in Influenza Vaccination Coverage Rates among Korean Cancer Survivors: Analysis of the Korea National Health and Nutrition Examination Survey III-VI
}

\author{
Ye-Seul Kim ${ }^{1,+}$, Jae-woo Lee ${ }^{1,+}$, Hee-Taik Kang ${ }^{1,2, *}$, Yonghwan Kim ${ }^{1}$, Hyo-Sun You' \\ 'Department of Family Medicine, Chungbuk National University Hospital, Cheongju, Korea \\ ${ }^{2}$ Department of Family Medicine, Chungbuk National University College of Medicine, Cheongju, Korea
}

Background: Few studies have reported on the trends in influenza vaccination rates among cancer survivors in Korea. We investigated these trends over a recent 11-year period (2005-2015).

Methods: Data were obtained from the Korea National Health and Nutrition Examination Survey (KNHANES) IIIVI (2005-2015). After excluding individuals younger than 40 years of age, the participants included 2,210 cancer survivors (861 men and 1,349 women). We categorized these study participants into four groups according to age and sex.

Results: The influenza vaccination rate decreased significantly among male cancer survivors, with rates of 74.8 , $64.7,56.2$, and $55.2 \%$ ( $\beta$ coefficient $=-0.257$, P for trend $<0.001$ ) in KNHANES III, VI, V, and VI, respectively. While influenza vaccination rates decreased significantly among older male cancer survivors ( $\geq 65$ years) ( $\beta$ coefficient $=-0.359$, $P$ for trend $=0.017$ ), they increased among older female cancer survivors ( $\beta$ coefficient $=0.375$, $P$ for trend $=0.011$ ) from 2005 to 2015.

Conclusion: Influenza vaccination rates in older cancer survivors significantly decreased in men but increased in women in successive KNHANES phases. Efforts are needed to improve immunization education and awareness, especially in older male cancer survivors.

Keywords: Influenza; Vaccination; Cancer Survivors; Population Growth

Received: September 18, 2018, Revised: November 7, 2018, Accepted: January 9, 2019

${ }^{*}$ Corresponding Author: Hee-Taik Kang https://orcid.org/0000-0001-8048-6247

Tel: +82-43-269-6301, Fax: +82-43-269-6675, E-mail: kanght0818@gmail.com

'These two authors contributed equally to this work (co-first authors). 


\section{INTRODUCTION}

Influenza virus infections cause diseases ranging from the common cold to severe respiratory illness, which can be fatal. ${ }^{1)}$ Influenza pandemics in the recent centuries include the Spanish flu in 1918, avian influenza A H5N1 in 1997 and 2003, and H1N1 swine flu in 2009. These worldwide influenza pandemics were caused by highly pathogenic influenza viruses and led to a significant number of fatalities. ${ }^{2)}$

Guidelines recommend vaccination of cancer survivors, who are at high risk for influenza infection. The Advisory Committee on Immunization Practices recommends routine annual influenza vaccination for all persons aged at least 6 months if vaccination is not contraindicated.) Additionally, the Infectious Diseases Society of America recommends annual inactivated influenza vaccination for patients aged $\geq 6$ months with hematological malignancies or solid malignant tumors. ${ }^{4)}$

According to Korean cancer statistics in 2015, ${ }^{5)}$ approximately 1.6 million people in Korea had a history of cancer diagnosis, and the 5 -year relative survival rates for cancer have steadily improved, from $41.2 \%$ in $1993-1995$ to $70.7 \%$ in $2011-2015$. According to previous studies in Korea, the influenza vaccination rate among cancer survivors ranged from $34.1 \%{ }^{6)}$ to $50 \%,{ }^{7)}$ indicating that only a small proportion of cancer survivors is vaccinated against influenza virus.

Although several studies have reported influenza vaccination rates among cancer survivors in Korea, none have reported trends in these rates according to age from nationwide data. Our study investigated recent 11-year trends in vaccination rates among Korean cancer survivors using data from the Korea National Health and Nutrition Survey (KNHANES) III (2005) to VI (2013-2015).

\section{METHODS}

\section{Study Population}

The KNHANES, which involves cross-sectional and nationally representative surveys, has been conducted by the Korea Centers for Disease Control and Prevention (KCDC) since 1998, following the National Health Promotion Act. The KNHANES has collected data on 23 households from each of the 192 districts as a probabilistic sample annually, surveying approximately 10,000 household members aged 1 year or older. To date, the KNHANES has been performed in six phases: KNHANES phase I (1998), II (2001), III (2005), IV (2007-2009), V (2010-2012), and VI (2013-2015). The sampling encompassed stratified, clustered, and systematic methods based on sex, age, and geographic area using household registries. To obtain information on health status, health behavior, socioeconomic demographics, and laboratory test results, the survey comprises three components: a health interview, a health examination, and a nutrition survey. Trained personnel conduct face-to-face interviews with participants. Participants may refuse to participate in this survey or may provide written consent. $^{8)}$

We used data from KNHANES III, IV, V, and VI. ${ }^{9)}$ After excluding subjects younger than 40 years of age, we included 2,210 cancer survivors (861 men and 1,349 women) and 51,704 non-cancer (22,952 men and 28,752 women) participants. Information on socio-demographic characteristics, such as age, sex, education, household income, and residential area, was gathered from self-reported questionnaires completed in each relevant survey period.

The Institutional Review Board of the KCDC approved this study and confirmed that informed consent was obtained.

\section{Definitions of Cancer Survivors, Vaccination, and Lifestyle Factors}

Cancer survivors were defined as individuals who reported having been diagnosed with any cancer by physicians, based on self-reported questionnaires.

Influenza vaccination was considered positive if participants answered "yes" to the question "Have you been vaccinated against influenza (seasonal flu) within the past year?"

Individuals who engaged in vigorous physical activity 3 or more days per week for at least 20 minutes per day or in moderate physical activity (including walking) 5 or more days per week for at least 30 minutes per day were classified as having "sufficient physical activity" (as per the International Physical Activity Questionnaire Research

Table 1. General characteristics of cancer survivors according to sex and KNHANES phase

\begin{tabular}{|c|c|c|c|c|c|}
\hline \multirow{2}{*}{ Characteristic } & \multicolumn{5}{|c|}{ KNHANES phase } \\
\hline & Total & III (2005) & IV (2007-2009) & V (2010-2012) & VI (2013-2015) \\
\hline Total (unweighted) & 2,210 & 448 & 470 & 619 & 673 \\
\hline Mean age (y) & $61.5 \pm 0.3$ & $63.4 \pm 1.4$ & $60.7 \pm 0.7$ & $60.7 \pm 0.6$ & $62.0 \pm 0.6$ \\
\hline Men (unweighted) & 861 & 197 & 173 & 225 & 266 \\
\hline Mean age (y) & $64.2 \pm 0.5$ & $67.2 \pm 2.0$ & $64.3 \pm 1.1$ & $63.2 \pm 1.0$ & $64.5 \pm 0.9$ \\
\hline 40-64 y (\%) & $47.1 \pm 2.3$ & $31.4 \pm 9.0$ & $47.4 \pm 4.8$ & $49.2 \pm 4.2$ & $47.0 \pm 3.7$ \\
\hline$\geq 65$ y $(\%)$ & $52.9 \pm 2.3$ & $68.6 \pm 9.0$ & $52.6 \pm 4.8$ & $50.9 \pm 4.2$ & $53.0 \pm 3.7$ \\
\hline Women (unweighted) & 1,349 & 251 & 297 & 394 & 407 \\
\hline Mean age (y) & $59.8 \pm 0.4$ & $61.7 \pm 1.5$ & $58.7 \pm 0.9$ & $59.2 \pm 0.7$ & $60.3 \pm 0.7$ \\
\hline 40-64 y (\%) & $64.1 \pm 1.6$ & $55.3 \pm 6.1$ & $63.8 \pm 3.6$ & $67.7 \pm 2.9$ & $62.8 \pm 2.7$ \\
\hline$\geq 65$ y $(\%)$ & $35.9 \pm 1.6$ & $44.7 \pm 6.1$ & $36.2 \pm 3.6$ & $32.3 \pm 2.9$ & $37.2 \pm 2.7$ \\
\hline
\end{tabular}


Table 2. Comparisons of general characteristics between cancer survivors and non-cancer controls

\begin{tabular}{|c|c|c|c|c|c|c|}
\hline \multirow{2}{*}{ Characteristic } & \multicolumn{3}{|c|}{ Men } & \multicolumn{3}{|c|}{ Women } \\
\hline & Cancer survivors & Non-cancer controls & P-value & Cancer survivors & Non-cancer controls & P-value \\
\hline Total no. & 861 & 22,952 & & 1,349 & 28,752 & \\
\hline Mean age (y) & $64.2 \pm 0.5$ & $54.7 \pm 0.1$ & $<0.001$ & $59.8 \pm 0.4$ & $56.7 \pm 0.1$ & $<0.001$ \\
\hline Age $(\%)$ & & & $<0.001$ & & & $<0.001$ \\
\hline $40-64$ y & $47.1 \pm 2.3$ & $80.4 \pm 0.4$ & & $64.1 \pm 1.6$ & $73.1 \pm 0.4$ & \\
\hline$\geq 65 y$ & $52.9 \pm 2.3$ & $19.6 \pm 0.4$ & & $35.9 \pm 1.6$ & $26.9 \pm 0.4$ & \\
\hline Monthly household income (\%) & & & $<0.001$ & & & 0.109 \\
\hline Lowest & $31.4 \pm 2.2$ & $17.8 \pm 0.4$ & & $28.0 \pm 1.5$ & $24.7 \pm 0.5$ & \\
\hline Middle-lowest & $26.4 \pm 2.1$ & $25.3 \pm 0.5$ & & $26.6 \pm 1.6$ & $25.7 \pm 0.4$ & \\
\hline Middle-highest & $19.7 \pm 1.8$ & $27.6 \pm 0.5$ & & $21.8 \pm 1.5$ & $24.1 \pm 0.4$ & \\
\hline Highest & $22.5 \pm 2.1$ & $29.4 \pm 0.6$ & & $23.6 \pm 1.6$ & $25.5 \pm 0.5$ & \\
\hline Marital status (\%) & & & 0.380 & & & 0.071 \\
\hline Single & $9.4 \pm 1.3$ & $8.2 \pm 0.3$ & & $29.1 \pm 1.6$ & $26.3 \pm 0.4$ & \\
\hline Married and not separated & $90.6 \pm 1.3$ & $91.8 \pm 0.3$ & & $70.9 \pm 1.6$ & $73.7 \pm 0.4$ & \\
\hline Education level (\%) & & & $<0.001$ & & & 0.006 \\
\hline Elementary school or less & $34.6 \pm 2.2$ & $20.4 \pm 0.4$ & & $43.9 \pm 1.7$ & $40.1 \pm 0.5$ & \\
\hline Middle school & $16.8 \pm 1.8$ & $15.4 \pm 0.4$ & & $17.2 \pm 1.2$ & $14.8 \pm 0.3$ & \\
\hline High school & $26.6 \pm 2.2$ & $35.0 \pm 0.5$ & & $26.8 \pm 1.6$ & $30.4 \pm 0.4$ & \\
\hline College or more & $22.1 \pm 2.0$ & $29.2 \pm 0.6$ & & $12.1 \pm 1.2$ & $14.7 \pm 0.4$ & \\
\hline Occupation $(\%)^{*}$ & & & $<0.001$ & & & $<0.001$ \\
\hline Office workers & $12.6 \pm 1.7$ & $24.6 \pm 0.5$ & & $7.6 \pm 1.0$ & $9.7 \pm 0.3$ & \\
\hline Manual workers & $35.3 \pm 2.2$ & $53.8 \pm 0.6$ & & $26.7 \pm 1.6$ & $38.6 \pm 0.5$ & \\
\hline Others & $52.2 \pm 2.3$ & $21.6 \pm 0.4$ & & $65.7 \pm 1.7$ & $51.7 \pm 0.5$ & \\
\hline Residence (\%) & & & 0.109 & & & 0.834 \\
\hline Urban & $74.6 \pm 1.9$ & $77.5 \pm 1.0$ & & $78.3 \pm 1.5$ & $78.0 \pm 0.9$ & \\
\hline Rural & $25.4 \pm 1.9$ & $22.5 \pm 1.0$ & & $21.7 \pm 1.5$ & $22.0 \pm 0.9$ & \\
\hline Sufficient activity $(\%)^{\dagger}$ & $52.5 \pm 2.8$ & $50.6 \pm 0.6$ & 0.511 & $44.7 \pm 2.0$ & $45.3 \pm 0.6$ & 0.793 \\
\hline Heavy alcohol-drinking $(\%)^{\ddagger}$ & $10.2 \pm 1.6$ & $21.5 \pm 0.4$ & $<0.001$ & $1.7 \pm 0.5$ & $3.3 \pm 0.2$ & 0.021 \\
\hline Current smoking (\%) & $20.4 \pm 2.0$ & $41.1 \pm 0.5$ & $<0.001$ & $3.1 \pm 0.6$ & $4.9 \pm 0.2$ & 0.027 \\
\hline Perceived stress symptoms $(\%)^{\S}$ & $22.8 \pm 1.9$ & $23.3 \pm 0.4$ & 0.815 & $28.6 \pm 1.5$ & $27.1 \pm 0.4$ & 0.357 \\
\hline Depressive mood (\%)" & $12.7 \pm 1.5$ & $11.5 \pm 0.3$ & 0.414 & $24.6 \pm 1.7$ & $20.5 \pm 0.4$ & 0.014 \\
\hline Chronic diseases (\%) $)^{n}$ & $52.8 \pm 2.3$ & $36.2 \pm 0.5$ & $<0.001$ & $44.5 \pm 1.7$ & $35.8 \pm 0.4$ & $<0.001$ \\
\hline \multicolumn{7}{|l|}{ Cancer type (\%) } \\
\hline Gastric & $30.1 \pm 2.1$ & & & $12.3 \pm 1.1$ & & \\
\hline Hepatic & $7.2 \pm 1.3$ & & & $0.8 \pm 0.3$ & & \\
\hline Colon & $17.9 \pm 1.8$ & & & $6.3 \pm 0.9$ & & \\
\hline Breast & & & & $21.4 \pm 1.4$ & & \\
\hline Cervix & & & & $20.2 \pm 1.4$ & & \\
\hline Lung & $5.4 \pm 1.0$ & & & $0.9 \pm 0.3$ & & \\
\hline Thyroid & $3.0 \pm 0.7$ & & & $17.2 \pm 1.3$ & & \\
\hline Others & $34.5 \pm 2.2$ & & & $21.0 \pm 1.4$ & & \\
\hline \multicolumn{7}{|l|}{ Duration since cancer diagnosis (\%) } \\
\hline$<2 y$ & $22.3 \pm 1.9$ & & & $13.8 \pm 1.2$ & & \\
\hline $2 y$ to $<5 y$ & $26.2 \pm 2.0$ & & & $27.2 \pm 1.6$ & & \\
\hline $5 y$ to $<10 y$ & $27.7 \pm 2.1$ & & & $24.0 \pm 1.5$ & & \\
\hline$\geq 10 y$ & $23.8 \pm 1.9$ & & & $34.9 \pm 1.7$ & & \\
\hline Age at cancer diagnosis (y) & $58.0 \pm 0.6$ & & & $52.0 \pm 0.7$ & & \\
\hline
\end{tabular}

Values are presented as mean or $\% \pm$ standard error. P-values were obtained based on comparisons of the means (for continuous variables) or frequencies (for categorical variables) between cancer survivors and individuals without a history of cancer.

*Occupation was classified as office workers (general managers, government administrators, professionals, and simple office workers), manual workers (clerk; service and sales workers; skilled agricultural, forestry, and fishery workers; persons who operate or assemble craft, equipment, or machines; and factory workers), and others (unemployed, housekeepers, and students). ${ }^{\dagger}$ Vigorous activity for at least 20 minutes $\geq 3 \mathrm{~d} /$ wk and moderate-intensity activity including walking for at least 30 minutes $\geq 5 \mathrm{~d} /$ wk. ${ }^{\ddagger}$ More than 7

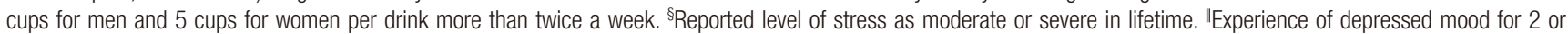
more continuous weeks during the previous year. "Hypertension; diabetes; liver cirrhosis; stroke; coronary heart disease; chronic renal disease; and lung diseases such as asthma, tuberculosis, and chronic obstructive pulmonary disease. 
Committee Guidelines for data processing and analysis of the International Physical Activity Questionnaire. ${ }^{10)}$ Men who consumed at least 7 cups and women who consumed at least 5 cups of alcohol at least twice per week were considered heavy alcohol drinkers. ${ }^{11)}$ Individuals who had smoked cigarettes during the relevant survey period were categorized as current smokers.

Education levels were divided into four groups: elementary school or less (education duration less than 6 years), middle school (education duration 6 to $<9$ years), high school (education duration 9 to $<12$ years), and college or more (education duration 12 years or more). Oc-

Table 3. Factors associated with influenza vaccination rates in cancer survivors in multivariate logistic regression analysis adjusted for all variables

\begin{tabular}{|c|c|c|c|c|c|c|}
\hline \multirow{2}{*}{ Variable } & \multicolumn{3}{|c|}{ Men } & \multicolumn{3}{|c|}{ Women } \\
\hline & All & $40-64$ y & $\geq 65 y$ & All & $40-64$ y & $\geq 65 y$ \\
\hline \multicolumn{7}{|l|}{ Age group (y) } \\
\hline $40-64$ & Reference & & & Reference & & \\
\hline$\geq 65$ & $2.52(1.10-5.79)$ & & & $2.55(1.46-4.44)$ & & \\
\hline \multicolumn{7}{|l|}{ Monthly household income } \\
\hline Lowest & Reference & Reference & Reference & Reference & Reference & Reference \\
\hline Middle-lowest & $1.25(0.58-2.69)$ & 5.18 (1.38-20.38) & $0.62(0.24-1.59)$ & $1.18(0.66-2.09)$ & $0.74(0.34-1.62)$ & $2.46(0.90-6.72)$ \\
\hline Middle-highest & $1.31(0.56-3.06)$ & $2.90(0.77-10.99)$ & $0.97(0.28-3.32)$ & $0.82(0.45-1.48)$ & $0.61(0.27-1.35)$ & $1.06(0.45-2.52)$ \\
\hline Highest & $1.30(0.49-3.45)$ & $2.76(0.70-10.91)$ & $1.76(0.35-8.77)$ & $0.97(0.54-1.75)$ & $0.62(0.27-1.31)$ & $2.55(0.76-8.60)$ \\
\hline \multicolumn{7}{|l|}{ Marital status (\%) } \\
\hline Single & $1.40(0.54-2.94)$ & $2.68(0.53-13.46)$ & $2.60(0.56-12.08)$ & $1.14(0.72-1.83)$ & $1.26(0.65-2.47)$ & $1.02(0.52-2.03)$ \\
\hline Married and not separated & Reference & Reference & Reference & Reference & Reference & Reference \\
\hline \multicolumn{7}{|l|}{ Education level (\%) } \\
\hline Elementary school or less & Reference & Reference & Reference & Reference & Reference & Reference \\
\hline Middle school & $1.26(0.54-2.94)$ & $2.51(0.66-9.53)$ & $0.96(0.29-3.18)$ & $0.94(0.56-1.56)$ & $0.91(0.50-1.66)$ & $0.95(0.37-2.42)$ \\
\hline High school & $0.90(0.45-1.79)$ & $2.51(0.66-9.53)$ & $0.76(0.26-2.21)$ & $0.84(0.48-1.45)$ & $0.82(0.44-1.56)$ & $1.33(0.36-4.90)$ \\
\hline College or more & $0.64(0.24-1.70)$ & $1.64(0.36-7.44)$ & $0.37(0.12-1.17)$ & $0.99(0.49-2.01)$ & $0.90(0.40-2.01)$ & $1.31(0.19-8.95)$ \\
\hline \multicolumn{7}{|l|}{ Occupation $(\%)^{*}$} \\
\hline Office workers & Reference & Reference & Reference & Reference & Reference & Reference \\
\hline Manual workers & $0.68(0.22-2.10)$ & $0.62(0.15-2.50)$ & $2.05(0.46-9.10)$ & $1.39(0.49-3.95)$ & $1.10(0.35-3.45)$ & NA \\
\hline Others & $0.68(0.22-2.13)$ & $0.29(0.07-1.16)$ & $3.91(0.99-15.45)$ & $1.50(0.56-4.03)$ & $1.33(0.46-3.82)$ & NA \\
\hline \multicolumn{7}{|l|}{ Residence } \\
\hline Urban & Reference & Reference & Reference & Reference & Reference & Reference \\
\hline Rural & $2.39(1.17-4.89)$ & $3.18(1.07-9.41)$ & $2.52(0.77-8.22)$ & $1.62(1.00-2.63)$ & $1.60(0.84-3.06)$ & $1.81(0.87-3.75)$ \\
\hline Heavy alcohol-drinking ${ }^{\dagger}$ (yes vs. no) & $0.65(0.23-1.80)$ & $1.05(0.28-3.97)$ & $0.55(0.15-2.02)$ & $0.18(0.02-2.11)$ & $0.14(0.01-1.93)$ & NA \\
\hline Current smoking (yes vs. no) & $0.60(0.28-1.29)$ & $0.57(0.18-1.80)$ & $0.55(0.21-1.48)$ & $0.52(0.12-2.19)$ & $0.72(0.19-2.73)$ & $0.72(0.04-0.87)$ \\
\hline Depressive symptoms ${ }^{\ddagger}$ (yes vs. no) & $0.83(0.37-1.86)$ & $1.77(0.38-8.24)$ & $0.70(0.26-1.88)$ & $0.62(0.39-0.99)$ & $0.68(0.38-1.20)$ & $0.61(0.31-1.20)$ \\
\hline Stress symptoms (yes vs. no) & $0.72(0.38-1.36)$ & $0.32(0.10-1.01)$ & $1.13(0.45-2.82)$ & $1.05(0.69-1.61)$ & $0.93(0.54-1.58)$ & $1.61(0.79-3.30)$ \\
\hline Chronic diseases"l (yes vs. no) & $1.13(0.65-1.96)$ & $1.02(0.44-2.39)$ & $0.72(0.36-1.46)$ & $1.10(0.72-1.68)$ & $1.04(0.62-1.73)$ & $1.15(0.53-2.49)$ \\
\hline \multicolumn{7}{|l|}{ Cancer type } \\
\hline Gastric & $0.43(0.13-1.36)$ & $0.21(0.04-1.15)$ & $0.78(0.22-2.76)$ & $0.65(0.10-4.30)$ & $0.48(0.04-6.03)$ & $0.99(0.16-6.03)$ \\
\hline Hepatic & $0.25(0.06-1.00)$ & $0.20(0.03-1.52)$ & $0.27(0.05-1.42)$ & $0.51(0.04-6.84)$ & $0.22(0.01-5.62)$ & NA \\
\hline Colon & $0.42(0.12-1.48)$ & $0.24(0.03-1.72)$ & $0.47(0.12-1.86)$ & $0.47(0.08-2.82)$ & $0.52(0.04-6.63)$ & $0.96(0.17-5.62)$ \\
\hline Breast & & & & $0.47(0.08-2.82)$ & $0.25(0.02-2.93)$ & $1.07(0.19-5.89)$ \\
\hline Cervix & & & & $0.54(0.09-3.26)$ & $0.30(0.03-3.55)$ & $1.22(0.21-6.95)$ \\
\hline Lung & Reference & Reference & Reference & Reference & Reference & Reference \\
\hline Thyroid & $0.07(0.01-0.36)$ & $<0.001$ & $0.25(0.04-1.72)$ & $0.53(0.09-3.27)$ & $0.30(0.03-3.51)$ & $3.28(0.35-30.44)$ \\
\hline Others & $0.60(0.19-1.91)$ & $0.33(0.06-1.80)$ & $0.82(0.21-3.23)$ & $0.48(0.08-3.27)$ & $0.34(0.03-3.88)$ & $0.67(0.14-3.25)$ \\
\hline \multicolumn{7}{|l|}{ Duration since cancer diagnosis (y) } \\
\hline$<2$ & Reference & Reference & Reference & Reference & Reference & Reference \\
\hline 2 to $<5$ & $1.06(0.50-2.29)$ & $0.49(0.13-1.90)$ & $2.38(0.95-5.98)$ & $0.96(0.53-1.75)$ & $0.94(0.47-1.86)$ & $0.66(0.18-2.35)$ \\
\hline 5 to $<10$ & $2.35(1.09-5.07)$ & $1.55(0.48-5.03)$ & $6.25(2.01-19.45)$ & $0.90(0.63-2.46)$ & $0.86(0.43-1.73)$ & $0.92(0.24-3.47)$ \\
\hline More than 10 & $2.92(1.03-8.27)$ & $8.20(1.88-35.81)$ & $1.93(0.54-6.95)$ & $1.25(0.63-2.46)$ & $1.40(0.64-3.07)$ & $0.85(0.23-3.22)$ \\
\hline Age at cancer diagnosis, 1 -year increase & $1.05(1.00-1.10)$ & $1.12(1.04-1.21)$ & $1.01(0.96-1.07)$ & $1.05(1.02-1.08)$ & $1.06(1.03-1.10)$ & $1.03(0.99-1.08)$ \\
\hline
\end{tabular}

Values are presented as adjusted odds ratio (95\% confidence interval).

*Occupation was classified as office workers (general managers, government administrators, professionals, and simple office workers), manual workers (clerk; service and sales workers; skilled agricultural, forestry, and fishery workers; persons who operate or assemble craft, equipment, or machines; and factory workers), and others (unemployed, housekeepers, and students). ${ }^{\dagger}$ More than 7 cups for men and 5 cups for women per drink more than twice a week. *Experience of depressed mood for 2 or more continuous weeks during the previous year. ${ }^{\circledR}$ Reported level of stress as moderate or severe in lifetime. "Hypertension; diabetes; liver cirrhosis; stroke; coronary heart disease; chronic renal disease; and lung diseases such as asthma, tuberculosis, and chronic obstructive pulmonary disease. 
cupational status was categorized as manual workers, office workers, and other: (1) manual workers: clerks, or service or sales workers; skilled workers in agriculture, forestry, or fisheries, operators or assemblers of vehicles, boats, planes or equipment or other machinery; and factory workers; (2) office workers: managers, professionals, and administrators; and (3) other: defined as unemployed, housekeepers, and students. Individuals who experienced depressive mood for 2 or more consecutive weeks during the previous year were defined as having depressive symptoms. Participants who reported their level of stress as moderate or severe were defined as having stress symptoms. Finally, chronic diseases included hypertension, diabetes, liver cirrhosis, stroke, coronary heart disease, chronic renal disease, and lung diseases such as asthma, tuberculosis, and chronic obstructive pulmonary disease.

\section{Statistical Analysis}

All data on continuous variables are presented as means \pm standard errors (SEs). Data on categorical variables are presented as percentages \pm SEs. All sampling and weight variables were stratified, and the SAS survey procedure was used for statistical analysis to account for the complex sampling design and to provide nationally representative prevalence estimates. Survey regressions and chi-square tests were used to compare cancer survivors and non-cancer controls. P-values for the trends in vaccination rates were calculated among the KNHANES phases by logistic regression analyses after setting the phase as the continuous variable. Statistical analyses were performed using SAS ver. 9.4 (SAS Institute Inc., Cary, NC, USA). All statistical tests were two-tailed and statistical significance was defined as P-values $<0.05$.

A. Men

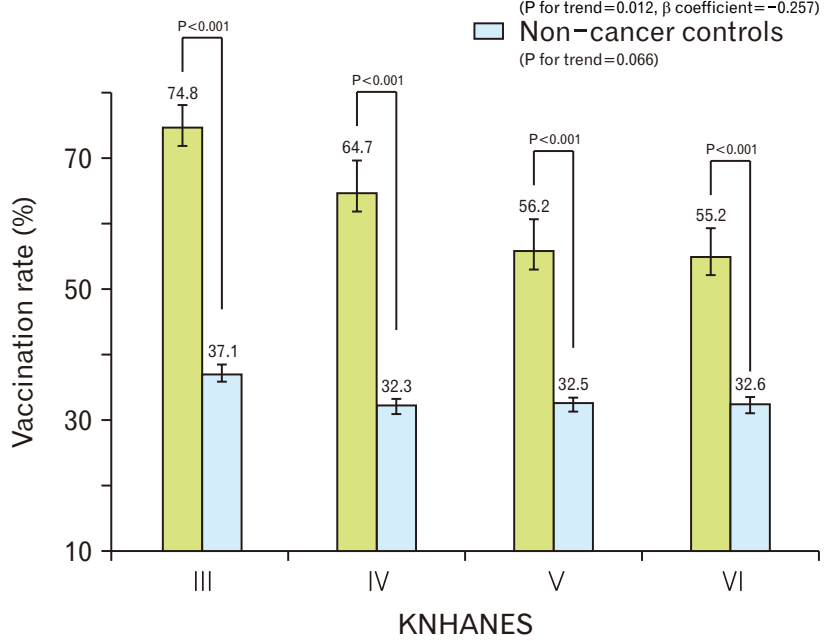

\section{RESULTS}

The final analysis included 2,210 cancer survivors (861 men and 1,349 women) (Table 1). The mean ages of all cancer survivors, men, and women were $61.5,64.2$, and 59.8 years, respectively.

Table 2 shows the general characteristics of cancer survivors and non-cancer controls. Cancer survivors were older than non-cancer controls for both sexes (64.2 versus 54.7 years in men and 59.8 versus 56.7 years in women). Cancer survivors were less educated, consumed smaller amounts of alcohol and tobacco, and had more chronic diseases than the non-cancer controls of both sexes. More male cancer survivors belonged to the low-income group. More female cancer survivors had a depressive mood. The three most common cancers were gastric $(30.1 \%)$, colon $(17.9 \%)$, and liver $(7.2 \%)$ cancer in men and breast (21.4\%), cervical (20.2\%), and thyroid (17.2\%) cancer in women.

Table 3 shows the factors associated with influenza vaccination. Older cancer survivors were more likely to have been vaccinated against influenza (odds ratio [OR], 2.52; 95\% confidence interval [CI], 1.10-5.79 in men and OR, 2.55; 95\% CI, 1.46-4.44 in women).

Survivors who lived in rural areas were more likely to have been vaccinated than were those living in urban areas. According to the duration after cancer diagnosis, significantly higher adjusted ORs (aORs) and 95\% CIs were observed for 5-10 and 10 years in men, at 2.35 (1.095.07) and 2.92 (1.03-8.27), respectively, whereas the aORs were not statistically significant in women. Age at cancer diagnosis was a central factor associated with influenza vaccination in both sexes. However, monthly household income, marital status, education levels, and occupation were not.

Figure 1 shows the influenza vaccination rates among cancer survivors and non-cancer control according to KNHANES phase. The influenza vaccination rates significantly decreased with successive KNHANES phases among male cancer survivors, at $74.8 \%, 64.7 \%$,

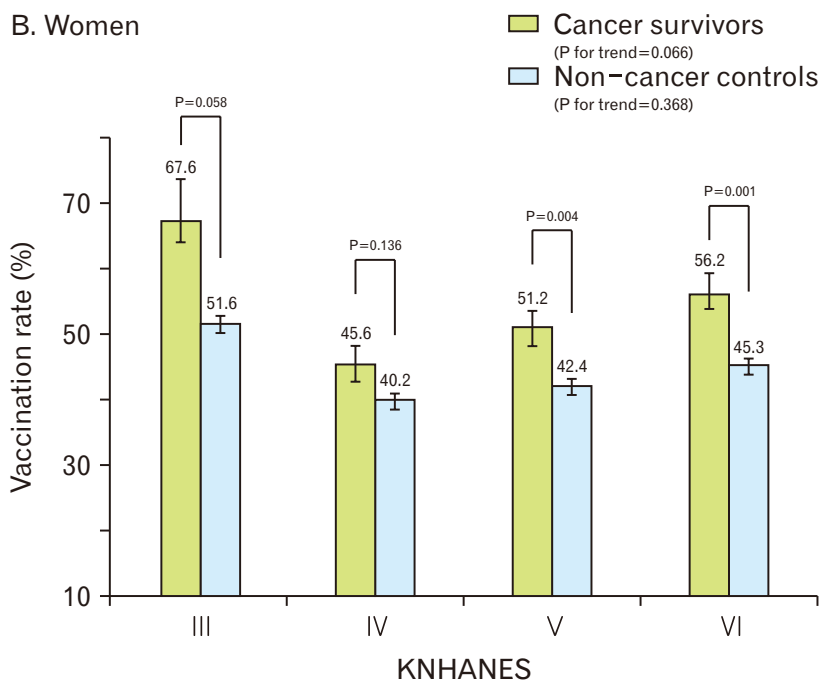

Figure 1. Trends for influenza vaccination rate among cancer survivors and non-cancer controls according to KNHANES phase. KNHANES, Korea National Health and Nutrition Examination Survey. 
$56.2 \%$, and 55.2\% ( $\beta$ coefficient $=-0.257$, $\mathrm{P}$ for trend $=0.012$ ) (Figure $1 \mathrm{~A})$, but did not significantly change in female cancer survivors, at $67.6 \%$, $45.6 \%, 51.2 \%$, and $56.2 \%$ from KNHANES III to VI, respectively (P for trend $=0.066$ ) (Figure 1B). Among non-cancer controls, there was no significant trend for either sex (all $\mathrm{P}$ for trend $>0.05$ ). The influenza vaccination rates of men were higher in cancer survivors than those in non-cancer controls across all KNHANES phases (all P-value <0.05). Those of women cancer survivors were significantly higher than those in non-cancer controls in KNHANES V and VI.

To investigate vaccination trends according to age, we stratified the participants into age groups of 40-64 and 65 years or older (Figure 2). Influenza vaccination rates significantly decreased with successive KNHANES phases in older male cancer survivors ( $\beta$ coefficient $=-0.359$, $P$ for trend=0.017), whereas the rates did not significantly change in older non-cancer controls. In women, the vaccination rates increased significantly in both older cancer survivors and non-cancer controls (all $\mathrm{P}$ for trend $>0.05$ ). In cancer survivors younger than 65 years of age, influenza vaccination rates did not significantly change with KNHANES phase in either sex (all $\mathrm{P}$ for trend $>0.05$ ).

\section{DISCUSSION}

This study investigated the trends in influenza vaccination among cancer survivors over the last 11 years. In this study, the influenza vaccination rates in cancer survivors were $74.8 \%, 64.7 \%, 56.2 \%$, and $55.2 \%$ in men and $67.6 \%, 45.6 \%, 51.2 \%$, and $56.2 \%$ in women from KNHANES III to VI, respectively. Those trends significantly decreased with KNHANES phase in male cancer survivors and did not change in female cancer survivors before stratification by age. Stafford et al. ${ }^{12)}$ reported an overall percentage of vaccine coverage among cancer survivors of $64 \%$, which was similar to our findings. Several Korean studies reported influenza vaccination coverage over $60 \%$ in a high-risk group and in cancer survivors, which were higher than that in the general population. ${ }^{13-15)}$ Morbidity and mortality rates are unusually high among individuals who are older and have risky medical conditions such as malignancies. Moreover, influenza-related respiratory infections in patients with cancer are often associated with costly hospitalizations, delays in potentially life-saving therapies, and death. ${ }^{16)}$ Korean health authorities provided free annual influenza vaccines to vulnerable populations such as infants and individuals older than 65 years

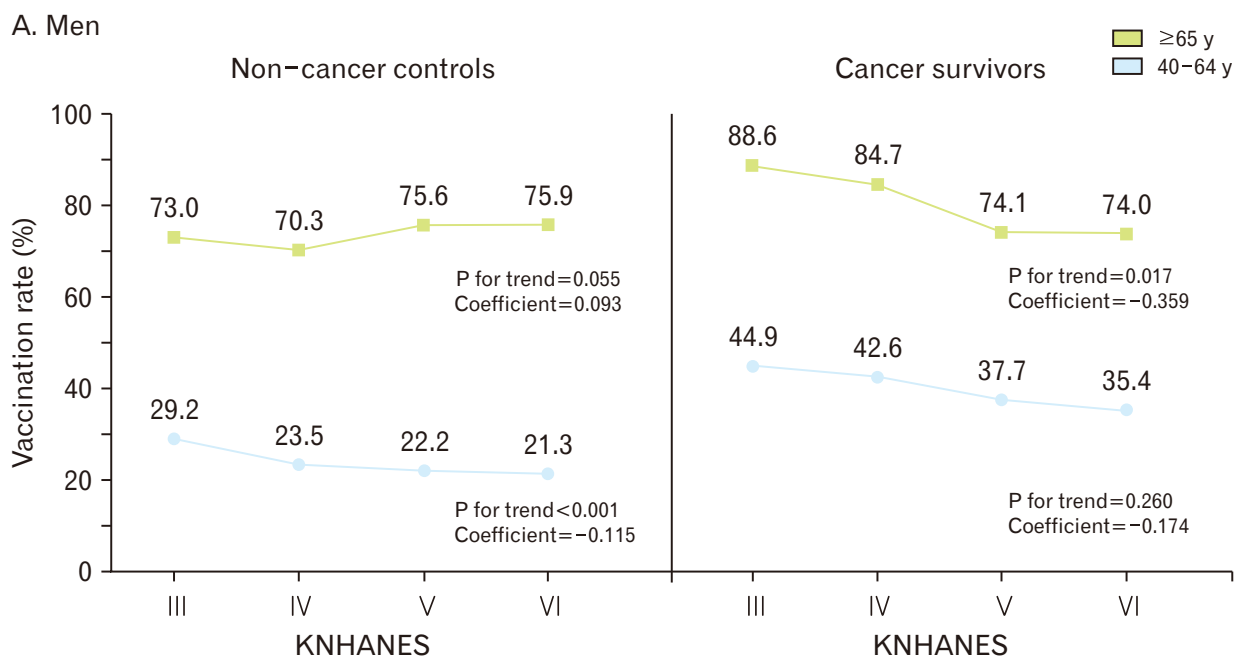

\section{B. Women}

Non-cancer controls

Cancer survivors $\square \geq 65$ y

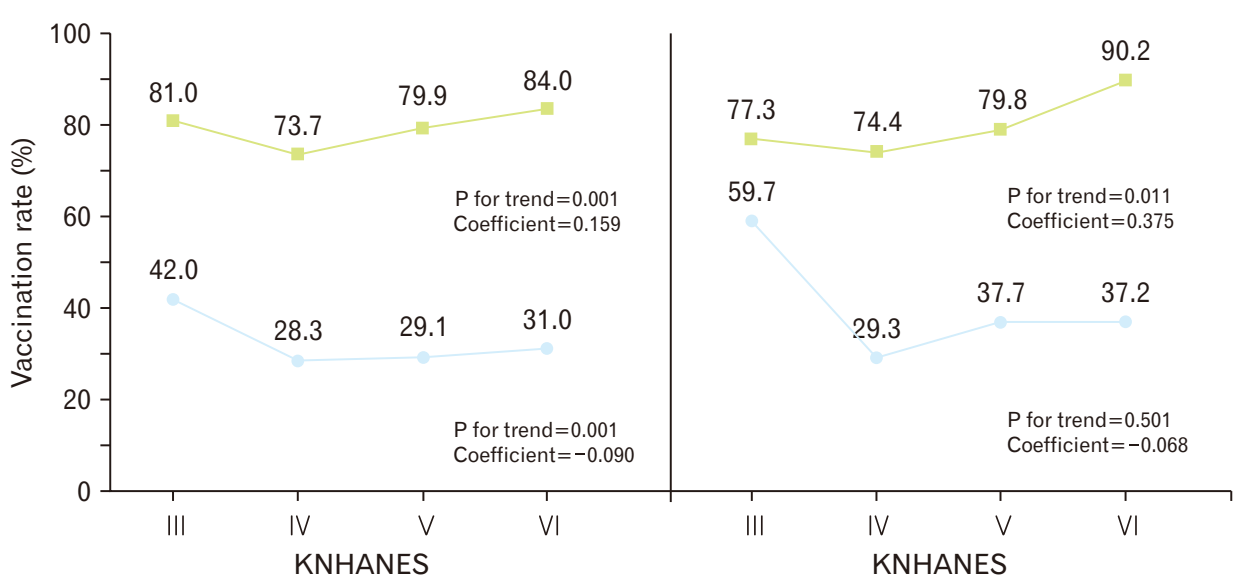

Figure 2. Trends for influenza vaccination rate among cancer survivors and non-cancer controls according to age group. KNHANES, Korea National Health and Nutrition Examination Survey. 
through the National Immunization Program (NIP). ${ }^{17)}$ Partly because of the NIP, the immunization rate against influenza among older Korean individuals is higher than that in other countries. ${ }^{18)}$

With the increase in the number of cancer survivors, the importance of managing chronic and infectious diseases in these individuals has also increased. Guidelines for cancer survivors recommend influenza; pneumococcal; and tetanus, diphtheria, and pertussis vaccinations according to the usual doses and schedules and human papillomavirus vaccine in survivors aged less than 26 years. ${ }^{19)}$

As reported by Choi et al., ${ }^{7}$ our analysis showed a higher influenza vaccination rate in older cancer survivors than that in younger cancer survivors. Moreover, the influenza vaccination rate increased significantly with KNHANES phase in older ( $\geq 65$ years) female cancer survivors. However, in older ( $\geq 65$ years) male cancer survivors, despite free immunization through NIP, the influenza vaccination rate significantly decreased with KNHANES phase in the present study.

In the United States, the Healthy People 2020 initiative has set a target influenza vaccination rate of up to $90 \%$ among high-risk adults, ${ }^{20)}$ while the European Union Council has recommended influenza vaccination rates of up to $75 \%$ among at-risk populations. ${ }^{21)}$ In Korea, there is no specific target rate for cancer survivors, but the KCDC has set a goal influenza vaccination rate of over $82.5 \%$ of the older population. ${ }^{9)}$ However, as indicated above, the influenza vaccination rate of cancer survivors in Korea has not reached either international or domestic targets. For example, in 2009, only $34.1 \%$ of cancer survivors had received an $\mathrm{H} 1 \mathrm{~N} 1$ vaccination and $53.5 \%$ had not considered the need for vaccination. ${ }^{6)}$ Another Korean study reported influenza vaccination rates of $50.3 \%$ and $31.5 \%$ in patients with and without cancer, respectively. ${ }^{7)}$ Despite higher vaccination coverage in cancer survivors than that in the general population, the influenza vaccination rate in younger cancer survivors should be increased. ${ }^{7,22)}$

The results of this study showed a significantly vaccination rate among cancer survivors than that of patients without a history of cancer. The most recent vaccination rate of female cancer survivors aged 65 years or older $(90.2 \%$ ) reached the goal of USA Healthy People 2020 and the KCDC, while rates of younger and older male cancer survivors were below the target. The vaccination rate was most closely related to age rather than socioeconomic status such as education level or occupation. In previous Korean studies, the relationship of education level and household income with vaccination rate was not consistent. ${ }^{23)}$

In part, higher vaccination rates in rural areas might have resulted from free immunization through the NIP, which began in 1997 in lowincome older populations and expanded to all adults over 65 years at public health centers from 2005 until 2014. Since 2015, the NIP for influenza has been expanded to private health sectors as well as public health centers. ${ }^{15)}$ The accessibility of public health centers is better in rural areas than that in urban areas. Even rural areas have good transportation systems and are highly connected. Distances between villages in Korea are not very great. Vaccination is also generally performed in groups.

Identification of barriers can result in achieving target vaccination rates and preventing severe influenza infection. The low vaccination rate in cancer survivors or patients with chronic diseases such as diabetes mellitus may be due to the low rate of vaccination at ages not included in the NIP. Because of the limitation of the survey questions, we could not confirm the reason for these results; however, it might be an epiphenomenon. The National Vaccine Advisory Committee recently suggested that health-care providers and patients lack knowledge and awareness about immunization. ${ }^{24)}$ However, based on the previous study, decreased vaccination rates in male patients $\geq 65$ years of age may be due to the lack of appropriate advice or information on the need for influenza vaccination or misconceptions about complications after influenza vaccination. ${ }^{25)}$ Additional research is needed. To increase the influenza vaccination rates for vulnerable at-risk populations such as cancer survivors, more efforts, such as education on vaccines by primary physicians and mass media are needed, especially for older men.

The present study has several limitations. First, we could not entirely exclude the possibility of reporting bias, as we utilized data from selfreported questionnaires for the diagnosis of cancer. Thus, the actual number of cancer survivors may have been underreported. Second, the KNHANES does not include people admitted to hospitals or nursing homes; therefore, the influenza vaccination rate among cancer survivors may have been underreported. Third, the cross-sectional study design was insufficient to conclude causality. As mentioned above, from 2015 onward, distribution of free vaccines was extended to private clinics. Expansion of the NIP is expected to result in changes in vaccination coverage, and further research is needed to identify related factors affecting vaccination rates.

Despite these limitations, the present study has several strengths. Our findings were derived from a large population-based sample with a complex survey design that aimed to generate estimates representative of the Korean population. To our knowledge, this is the first Korean study to examine trends in influenza vaccination coverage rates over the past decade using nationally representative data.

In conclusion, there were sex differences in influenza vaccination rates among cancer survivors. The coverage rate was significantly higher in older women and lower in older men in the KNHANES. Public disease prevention efforts are needed for male cancer survivors.

\section{CONFLICT OF INTEREST}

No potential conflict of interest relevant to this article was reported.

\section{ACKNOWLEDGMENTS}

This work was supported by the research grant of the Chungbuk National University Hospital in 2019.

\section{ORCID}

Ye-Seul Kim: https://orcid.org/0000-0002-1542-2367 
Jae-Woo Lee: https://orcid.org/0000-0003-1784-6494

Hee-Taik Kang: https://orcid.org/0000-0001-8048-6247

Yonghwan Kim: https://orcid.org/0000-0002-9965-675X

Hyo-Sun You: https://orcid.org/0000-0001-7254-5497

\section{REFERENCES}

1. Brinkhof MW, Spoerri A, Birrer A, Hagman R, Koch D, Zwahlen M. Influenza-attributable mortality among the elderly in Switzerland. Swiss Med Wkly 2006;136:302-9.

2. Barclay W, Openshaw P. The 1918 Influenza Pandemic: one hundred years of progress, but where now? Lancet Respir Med 2018;6:588-9.

3. Grohskopf LA, Sokolow LZ, Broder KR, Walter EB, Fry AM, Jernigan DB. Prevention and control of seasonal influenza with vaccines: recommendations of the advisory committee on immunization practicesUnited States, 2018-19 influenza season. MMWR Recomm Rep 2018; 67:1-20.

4. Rubin LG, Levin MJ, Ljungman P, Davies EG, Avery R, Tomblyn M, et al. 2013 IDSA clinical practice guideline for vaccination of the immunocompromised host. Clin Infect Dis 2014;58:e44-100.

5. Jung KW, Won YJ, Kong HJ, Lee ES; Community of Population-Based Regional Cancer Registries. Cancer statistics in Korea: incidence, mortality, survival, and prevalence in 2015. Cancer Res Treat 2018;50:30316.

6. Shin DW, Kim Y, Park JH, Cho J, Jho HJ, Yang HK, et al. Practices and predictors of $2009 \mathrm{H} 1 \mathrm{~N} 1$ vaccination in cancer patients: a nationwide survey in Korea. Influenza Other Respir Viruses 2012;6:e120-8.

7. Choi KH, Park SM, Lee K, Lee JH, Park JS. Influenza vaccination and associated factors among Korean cancer survivors: a cross-sectional analysis of the fourth \& fifth Korea National Health and Nutrition Examination Surveys. J Korean Med Sci 2014;29:1061-8.

8. Kweon S, Kim Y, Jang MJ, Kim Y, Kim K, Choi S, et al. Data resource profile: the Korea National Health and Nutrition Examination Survey (KNHANES). Int J Epidemiol 2014;43:69-77.

9. Korea Centers for Disease Control and Prevention National Immunization Program. Information for 2017 influenza vaccination. Cheongju: Korea Centers for Disease Control and Prevention; 2017.

10. International Physical Activity Questionnaire Research Committee. Guidelines for data processing and analysis of the International Physical Activity Questionnaire (IPAQ)-Short and Long Forms [Internet]. [place unknown]: International Physical Activity Questionnaire; 2005 [cited 2018 Sep 20]. Available from: https://www.academia. edu/5346814/Guidelines_for_Data_Processing_and_Analysis_of_the_ International_Physical_Activity_Questionnaire_IPAQ_Short_and_ Long_Forms_Contents.
11. Kang K, Sung J, Kim CY. High risk groups in health behavior defined by clustering of smoking, alcohol, and exercise habits: National Heath and Nutrition Examination Survey. J Prev Med Public Health 2010;43: 73-83.

12. Stafford KA, Sorkin JD, Steinberger EK. Influenza vaccination among cancer survivors: disparities in prevalence between blacks and whites. J Cancer Surviv 2013;7:183-90.

13. Kee SY, Lee JS, Cheong HJ, Chun BC, Song JY, Choi WS, et al. Influenza vaccine coverage rates and perceptions on vaccination in South Korea. J Infect 2007;55:273-81.

14. Oh CM, Won YJ, Jung KW, Kong HJ, Cho H, Lee JK, et al. Cancer statistics in Korea: incidence, mortality, survival, and prevalence in 2013. Cancer Res Treat 2016;48:436-50.

15. Seo J, Lim J. Trends in influenza vaccination coverage rates in South Korea from 2005 to 2014: effect of public health policies on vaccination behavior. Vaccine 2018;36:3666-73.

16. Avritscher EB, Cooksley CD, Geraci JM, Bekele BN, Cantor SB, Rolston $\mathrm{KV}$, et al. Cost-effectiveness of influenza vaccination in working-age cancer patients. Cancer 2007;109:2357-64.

17. Yun JW, Noh JY, Song JY, Chun C, Kim Y, Cheong HJ. The Korean influenza national immunization program: history and Present status. Infect Chemother 2017;49:247-54.

18. Yang HJ, Cho SI. Influenza vaccination coverage among adults in Korea: 2008-2009 to 2011-2012 seasons. Int J Environ Res Public Health 2014;11:12162-73.

19. Denlinger CS, Ligibel JA, Are M, Baker KS, Demark-Wahnefried W, Dizon D, et al. Survivorship: immunizations and prevention of infections, version 2.2014. J Natl Compr Canc Netw 2014;12:1098-111.

20. U.S. Department of Health and Human Services; Office of Disease Prevention and Health Promotion. Healthy people 2020. Rockville (MD): U.S. Department of Health and Human Services; 2000.

21. Nicoll A. A new decade, a new seasonal influenza: the Council of the European Union Recommendation on seasonal influenza vaccination. Euro Surveill 2010;15:19458.

22. Heo J, Chun M, Oh YT, Noh OK, Kim L. Influenza among breast cancer survivors in South Korea: a nationwide population-based study. In Vivo 2017;31:967-72.

23. Kang HS, Lee H, Kim MW. The correlates of influenza vaccination among Korean elderly men and women. J Korean Acad Community Health Nurs 2011;22:45-55.

24. National Vaccine Advisory Committee. Recommendations from the National Vaccine Advisory Committee: standards for adult immunization practice. Public Health Rep 2014;129:115-23.

25. Shin DW, Sunwoo S, Lee J. Management of cancer survivors in Korea. J Korean Med Assoc 2015;58:216-26. 